\title{
Physical and chemical house entry barriers to enhance odour-baited trapping for malaria control
}

\author{
R. Bokhorst \\ International Centre of Insect \\ Physiology \& Ecology \\ r.bokhorst@student.maastrichtuniversity.nl
}

\author{
A. Hiscox \\ Wageningen University and Research \\ Centre \\ solarmal@wur.nl
}

\begin{abstract}
Background: Odour-baited mosquito trapping is a promising new malaria intervention that lures, traps and kills mosquitoes. However, mosquitoes still bypass the trapping system and enter easily accessible African households through the open eaves. In this study, house screening and a spatial repellent were used as a physical and chemical house entry barrier to determine whether these interventions can protect people from malaria mosquitoes by decreasing mosquito house entry and simultaneously increasing odourbaited trapping efficacy.

Methods: In an experimental greenhouse in Kenya, two semi-field experiments were conducted. In the first experiment, three physical house adjustment interventions were tested against using no intervention: house screening (block); odour-baited trapping (pull) and both interventions (block-pull). In the second experiment, three chemical interventions were tested against using no intervention: a cotton band around the eaves impregnated with the spatial mosquito repellant Delta-undecalactone (push); odourbaited trapping (pull) and both interventions (push-pull). The effects of interventions were evaluated by comparing mean trap catches of the odour-baited trap outside, and mean mosquito house entry.

Results: In the first experiment (block-pull), the chance of a mosquito getting caught by an odour-baited trap more than doubled $(\mathrm{OR}=2.163)$ when complementing odour-baited trapping with house screening $(\mathrm{P}<0,001)$. All interventions significantly reduced mosquito house entry $(p<0,001)$, with the strongest protective effect for the combined intervention of house screening and odour-baited trapping $(\mathrm{OR}=0.10)$. The interventions of the second experiment did not have a significant effect on odour-baited trap catches $(p=0.584)$, nor mosquito house entry ( $p>0.172)$.
\end{abstract}


Discussion: This study shows that house screening could be a valuable addition to odourbaited mosquito trapping for malaria control and confirmed the strong effect of house screening on mosquito house entry. In contrast to other studies, a chemical barrier of Delta-undecalactone did not improve the protection against malaria mosquitoes. However, push-pull strategies might still be a viable alternative to house screening, since the absence of an effect might have been caused by an interaction of the intervention with the experimental environment.

\section{Keywords}

Malaria, house screening, odour baited trapping, spatial repellant, anopheles gambiae

\section{Introduction}

Malaria still tremendously burdens the population of Sub-Saharan Africa by causing for 600,000 out of 660,000 worldwide malaria deaths in 2010 (1). Malaria control methods such as indoor residual spraying (IRS) and insecticide treated bed nets (ITN's) are increasingly made available to reduce the burden of this disease. However, these strategies are under threat by growing insecticide resistance and outdoor biting behavior of malaria transmitting Anopheles mosquitoes (2). Inevitably, new malaria control tools need to be developed to interrupt malaria transmission (3). Odour-baited mosquito trapping technology could serve as a valuable complementary malaria control method. By mimicking components of human odour, the main cue that mosquitos use to locate their hosts, significant numbers of mosquitoes are prevented of reaching human hosts because they get lured into an odourbaited trap in which they die due to dehydration (4). Although the synthetic odour baits that lure malaria vectors are being further optimized $(5,6)$, mosquitoes are still capable of bypassing the trapping system hung outside a house (7). Mosquitoes mostly enter easily accessible households, and especially poorly constructed households with open eaves $(8,9)$. Therefore, it is relevant to look for interventions that could fill in this shortcoming.

An opportunity stems from blocking off house entry points using house screening. This intervention has already proven to significantly reduce indoor mosquito populations $(8,10)$. Moreover, by reducing the availability of the human hosts inside the house, the combination of house screening and odour-baited trapping (in this study referred to as block-pull interventions), could keep more mosquitoes outdoors with a physical barrier. Subsequently, these mosquitoes might be more likely to get attracted to, and caught by an odour-baited trap hung outside the house (7).

Another opportunity stems from mimicking push-pull interventions used in agriculture. 
These interventions draw away insects from crops that need protection, and simultaneously attract insects to places where they cause less harm or where they are captured in a trap. For malaria control, a 'push' stimulus from chemicals with spatial repellent properties could plausibly prevent Anopheles mosquitoes of accessing human host, and a 'pull' delivered by odour-baited mosquito traps would draw away, trap and kill mosquitoes.

The objective of this study is to analyse to what extent block-pull and push-pull strategies increase mosquito catches in odour-baited traps placed outdoors, while simultaneously minimizing mosquito house entry rates. Two semi-field experiments were designed to answer the following research questions:

1. To what extent do block-pull strategies using house screening, or push-pull strategies using a dUDL impregnated cotton band around the eaves increase the relative trapping efficacy of odour-baited mosquito traps placed outside a house?

2. To what extent do block-pull, or push-pull strategies, reduce mosquito house entry?

3. Do block-pull, or push-pull strategies, reduce mosquito house entry to a greater extent compared to using either block, pull, or push strategies as a single intervention method without an odour-baited trap

\section{Material and methods}

\section{Experimental environment}

The semi-field experiments were conducted in the MalariaSphere, an experimental greenhouse that facilitates all major life-history behaviors of mosquitoes, including hostseeking (11). It contains a house made out of mud walls and a thatch roof, with a bed with an un-impregnated bed net inside.

\section{Mosquitoes}

Anopheles gambiae sensu stricto (hereafter referred to as Anopheles gambiae) of the Mbita strain were used for the semi-field experiments. Mosquitoes were reared at the Tomas Odiambo campus of the International Centre of Insect Physiology and Ecology (icipe-TOC) at Mbita Point, Western Kenya. For each experiment, 200 female Anopheles gambiae in the age of 5-8 days were selected and caught 8 hours prior to initiation of the experiment. The mosquitoes were starved for 8 hours by secluding them from blood-carrying hosts and glucose solution.

\section{House screening (block)}

For block-pull interventions with house screening, one type of double-layered meshed 
netting material was used to cover all mosquitoes house entry points: the eaves and the crack above the door (figure 1). Additional gaps in the roof construction were sealed off by pushing folded screening material into the open spaces (figure 1b). The door was screened by fixing two layers of netting material on the outside of the door (figure $1 \mathrm{c}$ ).
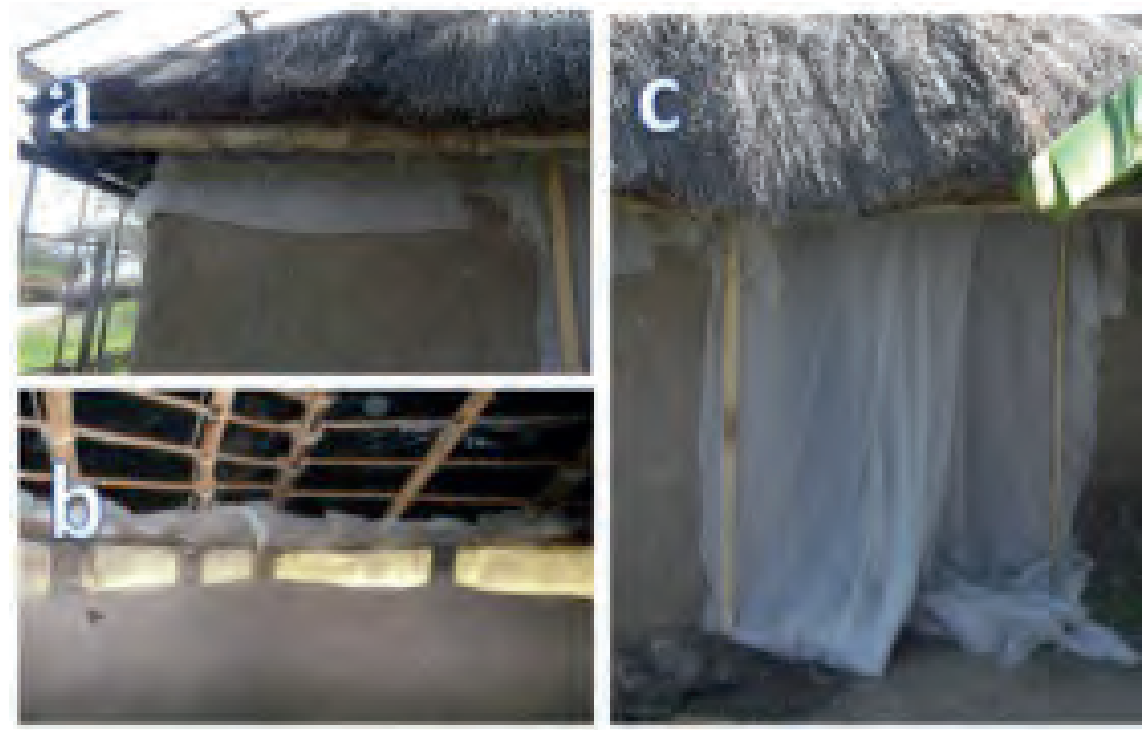

Figure 1. House screening applied for block-pull treatments. A visualizes how the eaves are screened and sealed off at the bottom by tying a rope around the wall of the house. B visualizes the eaves screening from inside the hut, as well as how additional gaps in the roof construction were sealed off using netting material. $C$ visualizes the door screening with two overlapping layers of screening material that were nailed into the wall with slats.

\section{Mosquito repellent (push)}

For the push-pull interventions, a $10 \mathrm{~cm}$ wide band of single-layered $100 \%$ cotton netting material was wound around the eaves (figure 2). For the control group and 'pullonly' interventions, the cotton was left un-impregnated. For 'push-only' and 'push-pull' interventions, an identical band impregnated with delta-undecalactone (dUDL) was fixed on the eaves. Medical examination cloves were worn while fixing both cotton bands around the eaves to prevent contamination of the material with human body odours. 

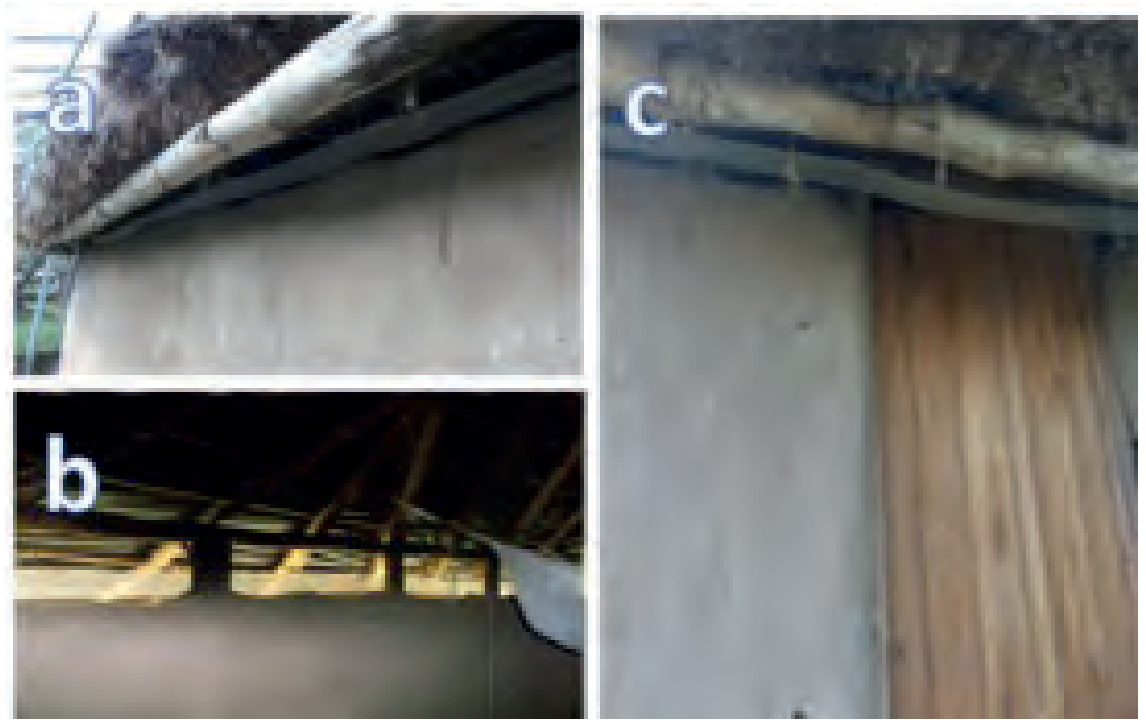

Figure 2. Band of cotton meshed netting material applied for push-pull treatments with dUDL repellent. A visualizes how the band is fixed around the eaves from the outside the house. B visualizes the cotton band from inside the hut. $C$ visualizes how the band is fixed above the door.

\section{Mosquito traps and odour-bait}

One unlit CDC miniature light trap (hereafter referred to as CDC trap), was used indoors to measure mosquito house entry. The light of the CDC trap remained unlit during all experiments to prevent bias from another stimulus attracting host-seeking mosquitoes. The CDC trap was hung inside the experimental hut at the foot-end of the bed. This position optimized indoor trap catches, because female Anopheles mosquitoes usually are attracted to the legs and feet (12). One mosquito Magnet-X (MM-X) trap was used for odour-baited mosquito trapping outside the experimental house (hereafter referred to as odour-baited trapping). The MM-X trap contained a counter flow mechanism dispersing a mixture of $\mathrm{CO}_{2}$ and synthetic odorants. Simultaneously, it sucked in nearby air, including lured host-seeking mosquitoes. The trap was placed outside by attaching it to the roof of the house with a synthetic cord.

The MM-X trap was baited with the Mbita blend, a mixture of synthetic odorants consisting of 3-methyl-1-butanol, tetradecanoic acid, ammonia solution, (S)-lactic acid. The Mbita blend was complemented with 1-butylamine. MB5 was dispensed via nylon strips and hung within the outlet of the MM-X trap, where the odorants were dispersed by the counter flow mechanism of the trap. The odour-blend was complemented by carbon 
dioxide (CO2), which was produced by mixing 17.5 grams of yeast with $250 \mathrm{ml}$ of molasses and 2 liters of water in an oil gallon (6).The baits were changed after six days of which the MM-X trap was part of an intervention. The yeast, molasses and water in the oil gallons were replaced each experimental night.

\section{Meteorological data}

For both experiments, temperature, humidity and rainfall were registered at, and extracted from, the icipe-TOC weather station located approximately 50 meters from the MalariaSphere. Temperature and humidity were averaged for each experimental night, whereas rainfall was registered as a binomial variable (yes/no). Meteorological conditions were assumed to be similar within the experimental environment.

\section{Experimental design}

The experiments were conducted in the course of October 2013 (block-pull) and November 2013 (push-pull). During all experiments, one local volunteer slept under an untreated bed net inside the house of the MalariaSphere. Each trial started at 20:00 hours, after switching on the mosquito traps, turning off the lights, and releasing 50 Anopheles gambiae from each corners of the MalariaSphere. The mosquitoes were released from four different corners to prevent the odour-baited MM-X trap or volunteer sleeping inside the house from having a relative advantage in proximity when mosquitoes are released from only one point of the MalariaSphere. At 06:30 hours experiments were terminated by switching off the traps, removing the capture bag from the CDC trap and taking the $M M-X$ trap in its entirety if used during the previous night. Both items were put in a freezer to kill the captured mosquitoes. The number of captured mosquitoes was counted later during the day. At 09:00 hours, indoor resting mosquitoes were counted by sight using a flashlight, and collected using an aspirator until no more mosquitoes could be found. These counts were added to the indoor CDC trap catches to calculate mosquito house entry per night. All semi-field experiments were executed for 6 successive nights. Randomization of interventions was regarded infeasible due to the possible residual effect of repellent impregnated cotton strips, and threats to the integrity of the netting material of when it needed to be removed and reused for multiple experimental night.

\section{Experiment 1: block-pull}

Experiment 1 estimated the effect of block-pull interventions on odour-baited trapping efficacy, as well on mosquito house entry rates. In order to estimate the effect of house screening on odour-baited trapping efficacy, the intervention combining odour-baited 
trapping and house screening (block-pull; figure $3 d$ ) was compared to using only odourbaited trapping (pull-only; figure $3 b$ ) on the number of mosquitoes caught outdoors by the MB5-baited MM-X trap. In order to estimate the effect of block-pull interventions on mosquito house entry rates, house screening (block-only; figure $3 b$ ), odour-baited trapping (pull-only; figure $3 c$ ), and the combination of these two interventions (block-pull) were compared to using no intervention (figure $3 a$ ) on their mean mosquito house entry rates. Furthermore, the mean rate of mosquito house entry by combining house screening with odour-baited trapping (block-pull) was assessed and compared to the reduction of using either trapping (pull only), or screening (block only), as a single intervention method.

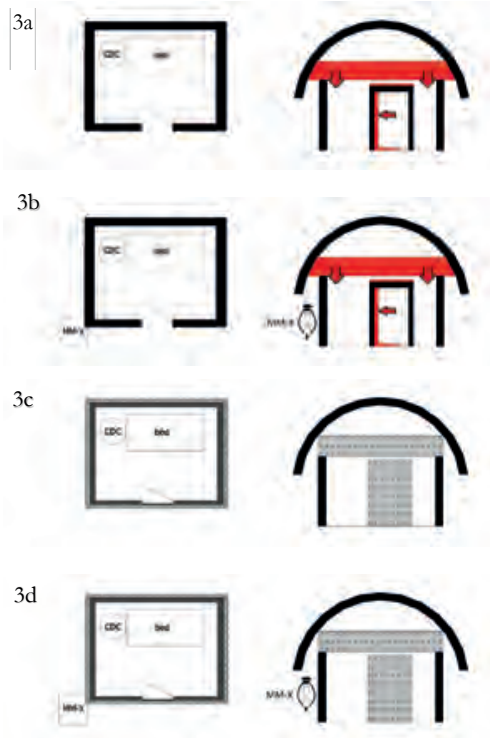

Figure 3. Block-pull treatments. $A$ is the control group without intervention, which a unlit CDC light trap indoors and a volunteer sleeping on the bed inside the house. $B$ is the intervention group with an additional $M M-X$ trap (pull only). $C$ is the house screening intervention (block only). $D$ is house screening complemented with an MM-X trap (block-pull). The house, excluding its roof, is displayed in bird view on the left, and the illustrations on the right display a cross-sectional view of the experimental house. The surface covered with screening materials colored grey, and filled up with crosses. The red areas display the "danger areas" through which mosquitoes are still physically capable of enter in the house.

\section{Experiment 2: push-pull}

Experiment 2 evaluated the effect of push-pull interventions on odour-baited trapping efficacy, as well on mosquito house entry rates. In order to measure the effect of placing 
a dUDL band on the efficacy of odour-baited mosquito trapping, the intervention combining odour-baited trapping and a dUDL band (push-pull; figure $4 \mathrm{~d}$ ) was compared to using only an odour-baited trapping (pull-only; figure 4 b) on the number of mosquitoes caught outdoors by the MB5-baited MM-X trap. In order to estimate the impact of blockpull interventions on mosquito house entry rates, the effect of placing a dUDL band (pushonly; figure 4 b), odour-baited trapping (pull-only; figure 4c), and the combination of these interventions (push-pull; figure 4d), were compared to using no intervention (figure 4a) on their mean mosquito house entry rates. Furthermore, the combination of odour-baited mosquito trapping and placing a dUDL band (push-pull) was estimated and compared against the reduction in mean house entry rates of using either trapping (pull only) or a dUDL band (push only) as a single intervention method.

$4 \mathrm{a}$
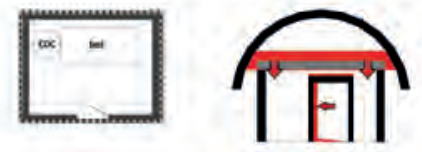

$4 \mathrm{~b}$
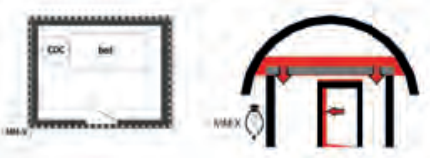

$4 c$
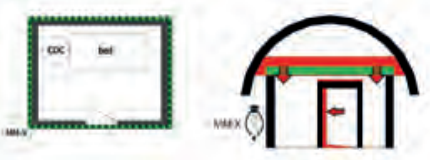

$4 d$

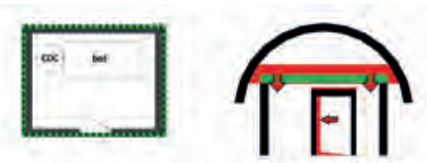

Figure 4. Push-pull treatments. A is the control group with an un-impregnated band of netting material tied around the eaves, with an unlit CDC light trap indoors and a volunteer sleeping on the bed inside the house. $B$ is the intervention group, which has an additional $M M-X$ trap compared to the control group (pull only). $C$ is the intervention group with a dUDL band (push only). D is the intervention group combining a dUDL band with an odor-baired MM-s trap outside the house (push-pull). The surface covered with netting material is filled up with the letter ' $R$ '. The un-impregnated band is colored grey, and the dUDL band is coloured green.

\section{Statistical analysis}

Normality tests were performed for both house entry rates and MM-X catches. Confidence intervals of mean house entry of some interventions included negative values. Therefore, 
tests assuming a normal distribution of the data were regarded as incorrect. Mean mosquito counts of house entry and outdoor odour-baited MM-X trap catches were compared between interventions using Generalized Linear Models (Poisson distribution, log link function) in IBM SPSS statistics software version 22.0. There was slight overdispersion in the data, but deviance was not significant, and Negative Binomial regression analysis did not improve model accuracy. Therefore, poisson regression was the accurate analysis for the data. Temperature averages during experimental nights were, if significant, added to multivariable models as covariates. Familywise error rates were corrected for using Bonferroni test for multiple comparisons, as the comparisons between interventions were dependent on comparisons of interventions with the control group (13). Additionally, proportions of the total number of captured mosquitos in odour-baited $M M-X$ catches outside the house versus indoor CDC trap catches were post-hoc compared against a 1:1 distribution using a one-sample proportions test with continuity correction in R-statistics version 3.0.1.

\section{Results}

\section{Experiment 1: Block-Pull}

Complementing odour-baited trapping by house screening (block-pull) significantly increased the efficacy of odour-baited trapping by increasing the chance of a mosquito being caught inside the odour-baited $M M-X$ trap more than twofold $(O R=2.163)$ and mean catch sizes by 49.34 mosquitoes (105.33\% ; P<0.001) compared to using odour-baited trapping as a single intervention method (push-only).

Table 1. Mean MM-X trap catches outside a house compared between block-pull combination treatment and pull-only treatment

\begin{tabular}{|c|c|c|c|c|}
\hline Treatment & $\begin{array}{l}\text { Mean } M M-X \\
\text { catch }\end{array}$ & $\begin{array}{l}\text { Difference mean } \\
M M-X \text { catch }(\%)\end{array}$ & $P$-value $(\alpha=0.05)$ & Odds Ratio (95\% CI) \\
\hline pull-only & 46.83 & - & - & - \\
\hline block-pull & 96.17 & $105.33 \%$ & $<0.001 *$ & $2.163(1.864-2.510)^{*}$ \\
\hline
\end{tabular}

All interventions significantly reduced mosquito house entry compared to no 'intervention' group. The strongest effect arose from combining odour-baited trapping and house screening (block-pull). This intervention reduced house entry risk by tenfold compared to the control group $(\mathrm{OR}=0.10)$ and mean house entry by 64.66 mosquitoes $(-90.02 \% ; \mathrm{P}<0.001)$. 
Table 2. House entry mean counts compared between block-pull treatments and control group

\begin{tabular}{l|lccc}
\hline Treatment & $\begin{array}{l}\text { House entry } \\
\text { mean }\end{array}$ & $\begin{array}{l}\text { Difference house entry } \\
\text { mean }(\%)\end{array}$ & $P$-value $(\alpha=0.05)$ & Odds Ratio (95\% CI) \\
\hline no interventionl & 71.83 & - & - & - \\
pull-only & 59.33 & $-17.40 \%$ & 0.008 & $0.826(0,718-0,950)$ \\
block-only & 11.33 & $-84.22 \%$ & $<0.001$ & $0.158(0.122-0.204)$ \\
block-pull & 7.17 & $-90.02 \%$ & $<0.001$ & $0.10(0.073-0.136)$ \\
\hline
\end{tabular}

Using odour-baited trapping as a complementary intervention method to house screening (block-pull) reduced the risk of a mosquito entering the house by 1.6 times $(O R=0.632)$ and mean house entry by 4.17 mosquitoes $(-36.76 \% ; P=0.019)$ compared to using house screening as a single intervention (block-only). House screening as a complementary intervention for odour-baited trapping (block-pull) reduced the risk of house entry by 8.26 times and mean house entry by 52.17 mosquitoes $(-87.92 \% ; \mathrm{P}<0.001)$ compared to using odour-baited trapping as a single intervention (pull-only). The marginal effect of both odour-baited trapping and house screening on house entry was lower when used as a complementary intervention rather than as single interventions (-8.34 mosquitoes for each intervention). However, the relative reduction in efficacy of the combined intervention (block-pull) was not significant compared to using each intervention separately, whereas the confidence intervals of the odds ratios of using odour-baited trapping or house screening as a single intervention method (block-only/push-only versus no intervention) and as a complementary intervention (block-pull versus block-only/push-only) were overlapping (table 2 and 3). Thus, the impact of each intervention was not influenced by the presence of the other.

Table 3. Effect of block-pull treatment on house entry compared to block-only and pull-only treatments

\begin{tabular}{l|lll}
\hline $\begin{array}{l}\text { Treatments } \\
\text { compared }\end{array}$ & $\begin{array}{l}\text { Difference house entry } \\
\text { mean }(\%)\end{array}$ & $\begin{array}{l}\text { P-value } \\
(\alpha=0.025) *\end{array}$ & Odds Ratio (95\% CI) \\
\hline $\begin{array}{l}\text { block-pull } v s . \\
\text { pull-only }{ }^{1}\end{array}$ & $-87.92 \%$ & $<0.001$ & $0.121(0.088-0.166)$ \\
$\begin{array}{l}\text { block-pull } v s . \\
\text { block-only }{ }^{2}\end{array}$ & $-36,76 \%$ & 0.019 & $0.632(0.432-0.926)$ \\
\hline $\begin{array}{l}\text { *adjusted for multiple comparisons using Bonferroni correction } \\
\text { 1 pull-only served as a reference group } \\
{ }^{2} \text { block-only served as a reference group }\end{array}$ &
\end{tabular}




\section{Experiment 2: Push-pull}

Complementing odour-baited trapping by a dUDL band (push-pull) did not have a significant effect on catch sizes of the odour-baited trap $(P=0.964)$.

Table 4. Mean MM-X trap catches outside a house compared between push-pull and pull-only treatments.

\begin{tabular}{l|llll}
\hline Treatment & $\begin{array}{l}\text { Total MM-X catch } \\
\text { outside }\end{array}$ & $\begin{array}{l}\text { Total CDC catch } \\
\text { indoor }\end{array}$ & Total catch & P-value \\
\hline pull-only 1 & 281 & 343 & 624 & 0.015 \\
pull-only 2 & 242 & 487 & 729 & $<0.001$ \\
\hline $\begin{array}{l}\text { I= experiment 1 } \\
2=\text { experiment 2 }\end{array}$ & & &
\end{tabular}

None of the interventions significantly reduced mosquito house entry compared to the control group. Differences between the combination of odour-baited trapping and a dUDL band, and using each intervention separately have not been calculated because the confidence intervals of the odds ratios of the interventions were overlapping and thus were insignificant.

Table 5. House entry means counts compared between push-pull treatments and control group

\begin{tabular}{l|lccc}
\hline Treatment & $\begin{array}{l}\text { Mean MM-X } \\
\text { catch }\end{array}$ & $\begin{array}{l}\text { Difference mean } \\
\text { MM-X catch }(\%)\end{array}$ & P-value $(\alpha=0.05)$ & Odds Ratio (95\% CI) \\
\hline pull-only & 40.33 & - & - & - \\
push-pull & 40.50 & $0.42 \%$ & $0.584^{*}$ & $0.950(0.792-1.140)^{*}$ \\
\hline
\end{tabular}

*adjusted for nightly variation in temperature

During both experiments, significantly more mosquitoes were caught in the CDC trap hung indoors compared to the amount of mosquitoes being caught in the odour-baited $M M-X$ trap outside the house $(P=0.015$ for experiment 1 , and $P<0.001$ for experiment 2$)$. The odour-baited trap caught $10 \%$, and $33 \%$ less mosquitoes than the CDC trap during the pull-only intervention in experiment 1 and experiment 2 , respectively. 
Table 6. Proportion of mosquitoes being caught outside the house versus inside the house.

\begin{tabular}{l|lllc}
\hline \multicolumn{1}{l}{ Treatment } & $\begin{array}{l}\text { House entry } \\
\text { mean }\end{array}$ & $\begin{array}{l}\text { Difference house entry } \\
\text { mean }(\%)\end{array}$ & P-value $(\alpha=0.05)$ & Odds Ratio (95\% CI) \\
\hline no intervention & 90 & - & - & - \\
pull-only & 82 & $-8.88 \%$ & $0.542 *$ & $1.019(0.889-1.167)^{*}$ \\
push-only & 85.17 & $-5.37 \%$ & $0.250^{*}$ & $1.086(0.944-1.250)^{*}$ \\
push-pull & 82.67 & $-8.15 \%$ & $0.172 *$ & $1.044(0.909-1.200)^{*}$ \\
\hline
\end{tabular}

*adjusted for nightly variation in temperature

\section{Discussion}

The results show that house screening could be a valuable addition to odour-baited trapping. Firstly, the chance of a mosquito being caught in an odour-baited trap outside a house more than doubled $(\mathrm{OR}=2.167$ ) when odour-baited trapping was complemented by house screening. Therefore, a block-pull intervention is likely to have a stronger impact on mosquito densities, biting intensity and even health indicators than using odour-baited trapping as a single intervention. Secondly, when a block-pull intervention is implemented, only one out of ten mosquitoes will succeed in entering a house $(O R=0,10)$. Thereby, the risk of indoor malaria transmission will strongly be reduced. This once again demonstrates the protective effect of house screening on mosquito house entry $(8,10)$. Thirdly, there is a significant additive effect of using house screening and odour-baited trapping in combination. This illustrates that house screening and odour-baited trapping do not pare down each other's effect when they are combined.

The block-pull experiment clearly demonstrated the complementary character of odourbaited trapping and house screening. However, implementing house screening on a large scale in houses with corrugated iron roofs and relatively unstable mud walls, the common house construction in malaria endemic areas, might be difficult. Moreover, the experimental house had a similar structure to houses found in traditional villages in the vicinity, and the provisional method of screening required large quantities of netting material to fill up the gaps between the roof and the poles supporting it. Thus, blockpull interventions require custom-made house screening, which likely is labor-intensive and costly. The second experiment measured an alternative strategy to reduce house entry and simultaneously increase odour-baited trapping efficacy. By creating a chemical house entry barrier that does not requires to entirely close off all house entry, push-pull interventions require less tailoring and craftsmanship to be implemented. However, experiment 2 shows that push-pull interventions are not yet optimized to a level that they 
could be ready for large scale implementation. None of the push-pull interventions had a significant effect on the efficacy of odour-baited mosquito trapping or mosquito house entry, nor did odour-baited trap catches outside the house increase when odour-baited trapping was complemented with a dUDL impregnated band around the eaves.

These findings were unexpected, as earlier experiments in the semi-field and field already demonstrated that the repellent effect of dUDL could significantly decrease mosquito house entry (14). However, the experimental design of this study could have altered the effect of push-pull interventions. Firstly, there was only a small amount of airflow through the eaves of the house in this experiment, because the screens enclosing the MalariaSphere disrupt wind speed, and the eaves are covered by the thatch roof that blocked the wind. Thereby, dUDL most likely only moleculary diffused throughout the whole space of the MalariaSphere (15). A while after placing the repellent band, the screenhouse could have had one homogeneous concentration of dUDL. Subsequently, mosquitoes would not have been able to escape continual exposure from the repellent whilst they were enclosed inside the MalariaSphere. Murlis \& Jones found that insects respond to instantaneous concentrations that are many-fold higher than mean concentrations (16). So, the homogeneous concentration of dUDL might not have triggered a behavioral response. In contrast, in the semi-field experiment conducted by Menger et al., dUDL was actively dispersing throughout the MalariaSphere using four MM-X traps at each corner of the house to dispense the dUDL (14). Thereby, plumes of dUDL were turbulently diffused throughout the air in concentrations higher than the surrounding area of the screenhouse. This likely formed a repellent barrier around the house and plausibly did trigger a behavioral response. Another plausible explanation is that the Anopheles' olfactory receptors might have become desensitized. Earlier research on longterm exposure to DEET already demonstrated that endured exposure to olfactory cues can lead to desensitization in Aedes aegypti species (17). If the same mechanism applies to Anopheles species, the malaria vector could have flown through the eaves without being irritated by the dUDL diffused from the cotton band as a result of long-term exposure to a homogeneous concentration of dUDL

Thus, under present conditions, it seems that dUDL is not repellent under all circumstances. More research is needed to clarify the requirements for effective implementation in order to further optimize push-pull strategies using mosquito repellents. For example, laboratory studies could test whether olfactory glands of mosquitoes need exposure to larger contrasts in concentration of dUDL to become responsive to the repellent. Additionally, wind tunnel experiments could simulate dispersion of dUDL throughout the experimental environment to determine how dUDL concentration changes when diffused at different wind velocities. 
An unexpected finding of this study is that the proportion of odour-baited trap catches outside the house was significantly lower than indoor CDC catches for pull-only interventions in both experiments $(\mathrm{P}=0.015$ for experiment 1 and $\mathrm{P}<0.001$ for experiment 2). More mosquitoes seem to be attracted to human odours from inside the house than to synthetic odours dispensed outside the house. Opposite results were found in a field trial with synthetic odours performed by Mukabana et al (6), in which was found that the synthetic odour blend was more attractive than a human being. In the latter study, attractiveness of synthetic odours dispensed by an $M M-X$ trap inside a house was compared to that of human odours coming from a human sleeping inside another house at a distance of minimally 25 meters. The contrasting results might be explained by the fact that in the present study, synthetic and human odours were competing, e.g. they were present within close proximity so that a mosquito could be able to detect the odour plumes emanating from both sources. Also in other studies with competing synthetic and human odours, human odours were more attractive $(7,18)$. Moreover, in experiments, which measured outdoor odour-baited catches against indoor catches using, synthetic versus synthetic odours (14), and human versus human odours (19), the proportion of mosquitoes caught in an odourbaited trap outside a house was significantly higher than the proportion caught inside. So, the outcomes of these experiments might indicate that mosquitoes are able to distinguish synthetic and human odours once they are competing in close spatial proximity. However, the contrast between indoor and outdoor catches in this study might also be attributed to inter-individual variation of humans in their relative attractiveness to mosquitoes, which can vary between being extremely attractive to even being repellent (20). The person sleeping inside the experimental house might have been relatively more attractive than volunteers of previous studies. Thereby, the proportion of mosquitoes caught indoors in the CDC trap places at his feet could have been relatively increased. An experimental design with competing synthetic and human odours, in a different environment using a random sample of volunteers that serve as a human attractant, could give more clarity on this important matter. In any case, these possible limitations to odour-baited trapping are strongly reduced when the intervention is complemented with house screening. The physical barrier of the screening material will make the entire spectrum of human hosts with different levels of attractiveness less accessible. This once again underlines the value of combining the interventions in a block-pull strategy.

\section{Future directions}

This study has shown that house screening could be a valuable addition to odour-baited mosquito trapping for malaria control. Additionally, it confirmed the strong effect of house 
screening on mosquito house entry. Furthermore, resource allocation for vector control could be improved by putting together two interventions that provide more benefits than when they are used as single interventions. Thereby, the results of this study provide an innovative tool that can facilitate current optimal vector control strategies, which is the main strategy of Integrated Vector Management (1). Based on the block-pull experiment of this study and previous findings of the effect of house screening on mosquito house entry $(8,10)$, large-scale implementation of house screening would be a recommendable extension of current malaria control practices in Kenya. Moreover, the findings of this study support integrating house screening in a block-pull strategy together with odourbaited mass trapping. Using this approach, mosquito population densities will likely further decrease by trapping malaria vectors, and preventing diversion of host-seeking Anopheles mosquitoes to other human hosts or cattle.

However, ongoing deficits in funding for malaria control (21) might limit the feasibility of these recommendations. In this case, odour-baited trapping could perhaps alternatively be implemented as a barrier of traps around a community, or around aquatic mosquito habitats (18). Yet, such a strategy would not be ideal, as odour-baited traps have been found to catch less mosquitoes when applied further away from inhabited areas (22). In addition, lack of ownership of the traps might cause problems to the maintenance and sustainability of the traps. Integration of odour-baited trapping systems with solar power could solve ownership problems. If governments would subsidize the purchase of solar powered trapping systems, people might be willing to buy the system because of the incentive to be provided with electricity in their homes. Logically, people will feel more responsible for their own investment. Moreover, caution with the implementation of house screening is advisable when there is a lack of funding. Pates and Curtis found that incomplete coverage with untreated bed nets diverts extra biting to people not having a bed net (23). The same is likely to happen on a larger scale, with some houses lacking screening within the community, most likely the poorest household that lack proper house screening in the community. Thereby, the poor become even more susceptible for malaria infections. Therefore, community-wide coverage of house screening should be guaranteed.

Results of push-pull interventions with dUDL that matched the protective effect of house screening in the semi-field (14) were not reproduced in this study. However, since the absence of an effect might have been caused by an interaction of the intervention with the experimental environment, push-pull interventions might still be a viable alternative to house screening. Moreover, house screening might even be combined with dUDL impregnation. This could possibly improve the sustainability of both interventions. 
A physical barrier could compensate for mosquitoes that become less susceptible to dUDL. Conversely, a chemical barrier could compensate for damage to the screening material. Therefore, it is recommendable to invest more funding in optimizing push-pull interventions to identify and address the flaws of this intervention. Subsequently, a field trial comparing house screening and a dUDL impregnated band around the eaves, and/or a combination of the two, would provide more information on which of these interventions would most strongly enhance odour-baited mosquito trapping while simultaneously decreasing mosquito house entry. Moreover, such a follow-up study would further facilitate the decision-making process of IVM by increasing knowledge on which of these promising intervention is most sustainable, socially acceptable and cost-effective. Finally, a strong combination of two interventions, which simultaneously reduce vector-human contact and kill mosquitoes that feed indoors and outdoors, could be the intervention that provides the additional push of malaria control towards malaria eradication.

\section{Role of the student}

Rens Bokhorst was an undergraduate student in Health Sciences and did a voluntary research intern at the SolarMalaria project, a collaboration of the International Centre of Insect Physiology \& Ecology (ICIPE) and Wageningen University \& Research Centre (WUR). The research question and study design were constructed on the intern's own initiative, with support of Dr. Richard Wolfgang Mukabana and Dr. Collins Mweresa of ICIPE. Both experiments were conducted by the intern, with support of Bruno Otieno and Anthony Kibet of the local SolarMal staff. In the course of the experiments and write up of the study, Dr. Alexandra Hiscox and Msc. David Menger from the medical entomology department of WUR supported the intern in completing the experiments and writing up the thesis.

\section{Acknowledgements}

I want to thank David Menger, Alexandra Hiscox and Willem Takken of Wageningen University and Research Centre, as well as Collins Mweresa and Richard Mukabana of ICIPE for their contributions to the design, interpretation and write-up of this study. Additionally, I want to thank the whole SolarMal project staff for receiving me at ICIPEin Mbita, as well as helping me to obtain and use the materials needed to conduct my experiments. Finally, I want to thank Jane Alaii, Bart van den Borne and Ree Meertens for facilitating my stay at a foreign institute as a Maastricht University student. 


\section{References}

1. WHO. World malaria report. 2012 [cited September 242013.

2. Takken, W. and B.G. Knols, Malaria vector control: current and future strategies. Trends in parasitology, 2009. 25(3): p. 101-104.

3. Control, m.C.G.o.V., A research agenda for malaria eradication: vector control. PLoS medicine, 2011. 8(1): $\mathrm{p}$. e1000401.

4. Okumu, F.O., et al., Development and field evaluation of a synthetic mosquito lure that is more attractive than humans. PloS one, 2010.5(1): p. e8951.

5. Hiscox, A., et al., The SolarMal Project: innovative mosquito trapping technology for malaria control. Malaria J, 2012. 11: p. O45.

6. Mukabana, W.R., et al., A novel synthetic odorant blend for trapping of malaria and other African mosquito species. Journal of chemical ecology, 2012. 38(3): p. 235-244.

7. Jawara, M., et al., Optimizing odor-baited trap methods for collecting mosquitoes during the malaria season in The Gambia. PLoS One, 2009. 4(12): p. e8167.

8. Lindsay, S., et al., Changes in house design reduce exposure to malaria mosquitoes. Tropical Medicine \& International Health, 2003. 8(6): p. 512-517.

9. Lwetoijera, D.W., et al., A need for better housing to further reduce indoor malaria transmission in areas with high bed net coverage. Parasit Vectors, 2013. 6: p. 57.

10. Kirby, M.J., et al., Effect of two different house screening interventions on exposure to malaria vectors and on anaemia in children in The Gambia: a randomised controlled trial. The Lancet, 2009. 374(9694): p. 998-1009.

11. Knols, B.G., et al., MalariaSphere: A greenhouse-enclosed simulation of a natural Anopheles gambiae (Diptera: Culicidae) ecosystem in western Kenya. Malaria journal, 2002.1(1): p. 19.

12. Mboera, L., et al., Short report: Influence of centers for disease control light trap position, relative to a humanbaited bed net, on catches of Anopheles gambiae and Culex quinquefasciatus in Tanzania. The American journal of tropical medicine and hygiene, 1998. 59(4): p. 595-596.

13. Howell, D., Statistical methods for psychology. 2012: Cengage Learning.

14. Menger, D.J., et al., A push-pull system to prevent house entry of malaria mosquitoes. Malaria journal, 2014.13

15. Murlis, J., J.S. Elkinton, and R.T. Carde, Odor plumes and how insects use them. Annual review of entomology, 1992. 37(1): p. 505-532

16. Murlis, J. and C. Jones, Fine-scale structure of odour plumes in relation to insect orientation to distant pheromone and other attractant sources. Physiological Entomology, 1981. 6(1): p. 71-86.

17. Stanczyk, N.M., et al., Aedes aegypti mosquitoes exhibit decreased repellency by DEET following previous exposure. Plos one, 2013. 8(2): p. e54438.

18. Okumu, F.O., et al., Potential benefits, limitations and target product-profiles of odor-baited mosquito traps for malaria control in Africa. PLoS One, 2010. 5(7): p. e11573.

19. Smallegange, R.C., et al., Sugar-fermenting yeast as an organic source of carbon dioxide to attract the malaria mosquito Anopheles gambiae. Malar J, 2010. 9(292): p. 10.1186

20. Verhulst, N.O., et al., Composition of human skin microbiota affects attractiveness to malaria mosquitoes. Plos one, 2011. 6(12): p. e28991.

21. UNAID. Delivering results toward ending AIDS, Tuberculosis and Malaria in Africa. African Union accountability report on Africa-G8 partnership commitments 2013. 2013 [cited 2013 December 4, 2013].

22. Qiu, Y.T., et al., Attractiveness of $M M-X$ traps baited with human or synthetic odor to mosquitoes (Diptera: Culicidae) in The Gambia. Journal of medical entomology, 2007. 44(6): p. 970.

23. Pates, H. and C. Curtis, Mosquito behavior and vector control. Annu. Rev. Entomol., 2005. 50: p. 53-70. 